\title{
Myocardial function and perfusion assessment with exercise stress cardiovascular magnetic resonance using an MRI-compatible treadmill in patients referred for stress SPECT
}

\author{
Paaladinesh Thavendiranathan ${ }^{1,2^{*}}$, Jennifer Dickerson ${ }^{2}$, Debbie Scandling ${ }^{2}$, Vijay Balasubramanian², Nathan Hall ${ }^{2}$,
} Eric Foster ${ }^{2}$, John W Arnold ${ }^{2}$, Michael Pennell ${ }^{2}$, Orlando P Simonetti ${ }^{2}$, Subha V Raman ${ }^{2}$

From 15th Annual SCMR Scientific Sessions

Orlando, FL, USA. 2-5 February 2012

\section{Background}

Exercise stress cardiac magnetic resonance (CMR) has recently become feasible with the development of a fully MRI-compatible treadmill system along with improvements in imaging techniques. The utility of this setup has not been systematically compared with nuclear perfusion imaging. The study objective was to evaluate the accuracy and prognostic value of exercise stress CMR with a treadmill placed immediately next to the MRI scanner table in patients referred for treadmill stress nuclear perfusion imaging.

\section{Methods}

39 patients (29 males) aged 33-78 (mean $54.3 \pm 12.4$ years) underwent a single treadmill stress study combined with CMR and single photon emission computed tomography (SPECT) imaging. After rest Tc99m SPECT imaging and resting CMR cine imaging, Bruce protocol stress was performed using a fully MRI-compatible treadmill placed adjacent to the scanner table. 12-lead ECG monitoring was performed throughout. At peak stress, Tc99m was injected and allowed to circulate for at least 90 seconds. The patient then quickly returned to the prior position in the magnet for post-exercise cine and perfusion imaging. The patient was then brought out of the magnet for recovery monitoring. After adequate recovery, the patient was sent back into the magnet for recovery cine and resting perfusion imaging, followed by late gadolinium enhancement imaging.

${ }^{1}$ Cardiovascular Medicine, Cleveland Clinic Foundation, Cleveland, $\mathrm{OH}$, USA Full list of author information is available at the end of the article
After CMR, each patient returned to the adjacent nuclear lab to complete stress SPECT imaging. Images from each modality were reviewed by 3 reviewers blinded to the results of the other modality (Figure 1). Coronary angiography or gated coronary computed tomography was performed in a subset of patients. Luminal stenosis $\geq 70 \%$ was considered to be significant coronary artery disease (CAD).

\section{Results}

Exercise time averaged 10.0 \pm 2.9 minutes. Exercise ECG was interpretable during all stages of exercise in $95 \%$ of the patients. Stress cine imaging commenced on average at $28 \pm 5 \mathrm{sec}$ following end of exercise, and stress function and perfusion were completed by $64 \pm 7 \mathrm{sec}$, vs. previously reported $42 \pm 5 \mathrm{sec}$ and $88 \pm 8 \mathrm{sec}$, respectively, using a partially modified treadmill in the corner of the scanner room. Agreement between SPECT and CMR for the detection of ischemia and scar was moderate $(\kappa=0.56)$. Accuracy for detection of significant CAD in the 21 patients who had coronary angiography was $21 / 21$ for CMR and $17 / 21$ for SPECT $(\mathrm{p}=0.13)$. Follow up at 8 12 months indicated excellent prognosis, with all patients with negative CMR or SPECT having no coronary events.

\section{Conclusions}

Exercise stress CMR using an MRI-compatible treadmill to assess wall motion and perfusion was feasible, accurate, and had good prognostic value in patients with suspected ischemic heart disease. Larger-scale, multicenter studies are needed to confirm our initial experience 


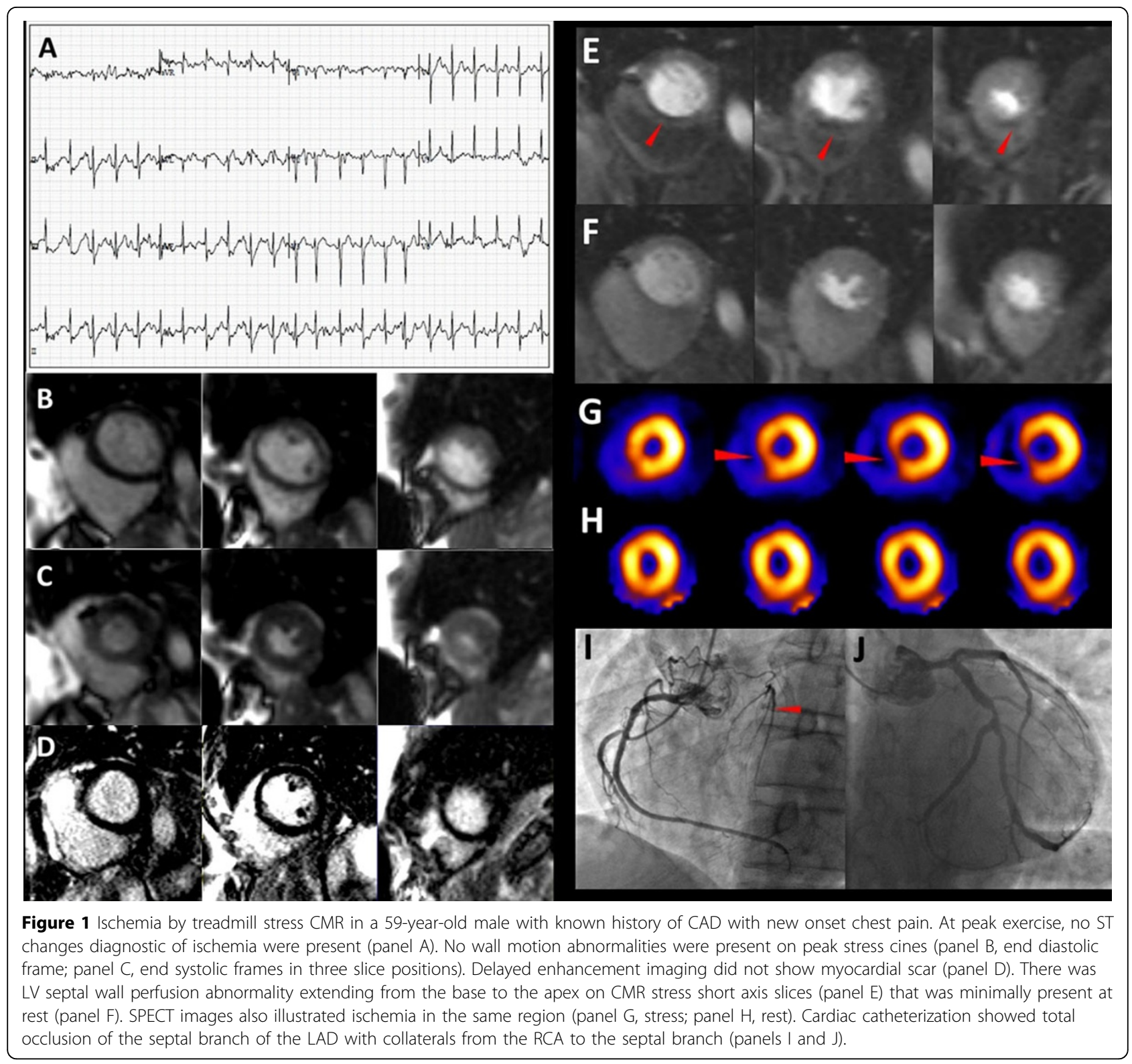

that suggests comparable accuracy of exercise CMR vs. SPECT to identify myocardial ischemia and infarct scar due to CAD.

\section{Funding}

National Institute of Health (NIH).

\section{Author details}

'Cardiovascular Medicine, Cleveland Clinic Foundation, Cleveland, OH, USA.

${ }^{2}$ Cardiovascular Medicine, The Ohio State University, Columbus, OH, USA.

Published: 1 February 2012
doi:10.1186/1532-429X-14-S1-P1

Cite this article as: Thavendiranathan et al: Myocardial function and perfusion assessment with exercise stress cardiovascular magnetic resonance using an $\mathrm{MRI}-$-compatible treadmill in patients referred for stress SPECT. Journal of Cardiovascular Magnetic Resonance 2012 14(Suppl 1):P1. 